\title{
Promoción de lectura en la universidad: Consideraciones pedagógicas
}

\author{
Yudit Rovira Álvarez ${ }^{a}$, Ernesto López-Calichs ${ }^{b}$ \& Alina Jiménez-Morejón ${ }^{a}$ \\ ${ }^{a}$ Dirección de Extensión Universitaria, Universidad “Hermanos Saíz Montes de Oca”, Pinar del Río, Cuba, judy@upr.edu.cu, alina72@upr.edu.cu \\ ${ }^{b}$ Centro de Estudios de Ciencias de la Educación Superior, Universidad “Hermanos Saíz Montes de Oca”, Pinar del Río, Cuba, ernest@upr.edu.cu
}

\begin{abstract}
Resumen - El presente artículo tiene como centro al proceso formativo en la universidad desde la promoción de la lectura, como respuesta a las necesidades de desarrollo cultural de la comunidad universitaria. El problema de la investigación estuvo dado en: ¿Cómo desarrollar el proceso de formación para la promoción de la lectura planificado, sistémico y sobre bases científicas, para el perfeccionamiento de la formación cultural de los estudiantes universitarios? La investigación transitó, mediante métodos teóricos como el Histórico Lógico, la Modelación , el Sistémico estructural así como empíricos y estadísticos. Como aporte teórico, se fundamentó una concepción pedagógica lo que permitió identificar tres etapas por las que transcurre, así como los principios que regulan su funcionamiento.
\end{abstract}

Palabras clave: universidad, extensión universitaria, formación, lectura, promoción, promoción de lectura.

Recibido: 29 de Abril de 2017. Revisado: 4 de Julio de 2017. Aceptado: 19 de Julio de 2017.

\section{Promotion of reading in the university: Pedagogical considerations}

Abstract - The present article has as center to the formative process in the university from the promotion of the reading, like answer to the needs of cultural development of the university community. The research problem was given in: How to develop the training process for the promotion of planned, systemic and scientific-based reading for the improvement of the cultural training of university students? The research carried out, through various research methods, the initial diagnosis of the training process for the promotion of reading and the development of the theoretical framework. As a theoretical contribution, a pedagogical conception on the method of cultural promotion as part of the university extension, which allowed three stages as well as the principles that regulate its operation.

Keywords: university, university extension, training, reading, promotion, reading promotion

\section{Introducción}

En el presente artículo se abordan algunas consideraciones teóricas devenidas de la fundamentación de una concepción pedagógica de formación para la promoción de lectura en la universidad y direccionado desde las particularidades que posee el proceso de extensión universitaria para su implementación, es decir, constituir este un proceso eminentemente formativo y poseer un método propio, la promoción sociocultural.

Referirse a la formación para la promoción de lectura supone dar cuenta no solo de aspectos específicamente vinculados con la formación universitaria, sino también poner de relieve sus dimensiones, coincidentes con las de un proceso formativo: curricular y extracurricular, su sistema de principios, así como las etapas por las que transcurre.

Se argumentan cuestiones culturales, pedagógicas y metodológicas que operarán, sin duda, como presupuestos de trabajo de los aspectos antes mencionados. En este sentido, se podría sostener que la Educación Superior precisa considerar las potencialidades de la extensión como proceso formativo y como función universitaria para la implementación de innovaciones desde la lectura y su promoción.

\subsection{El proceso de formación para la promoción de lectura se sustenta en la extensión universitaria y en la promoción sociocultural como método, desde las dimensiones curricular y extracurricular}

La importancia de la lectura en la universidad ha sido señalada por [1] que expresa las instituciones de educación superior deben formar lectores activos para desarrollar en ellos las capacidades que les permitan hacer de la información el aprendizaje, los conocimientos y las innovaciones, soluciones dirigidas hacia la elevación del bienestar social de todos.

En sus reflexiones [2] acerca de la promoción de lectura ha alertado sobre las carencias de los sujetos y la ineficacia del sistema educativo, las falencias de los docentes y la pauperización de la cultura letrada.

Por otra parte [3] expresa que en la enseñanza superior el estudiante debe tener protagonismo hacia su propia formación, y saber que en este tipo de enseñanza encontrará exigencias mayores en sus estudios, por lo que con conciencia de esa nueva situación debe autogestionar su formación.

En ese orden de ideas [4] plantea la importancia de la lectura como herramienta para el acceso a la información, lo que es un aspecto esencialmente ligado a la enseñanza universitaria.

La evolución de la educación superior cubana y de la propia extensión universitaria, reafirman el significado de una concepción formativa en este sentido.

Hablar de una concepción de formación desde el proceso extensionista permite adentrarse en un complejo tejido subjetivo y social que se va tramando de formas muy específicas en cada contexto y en cada sujeto, sin negar los 
históricos legados que se continúan perpetuando de generación en generación con los necesarios círculos evolutivos, que denotan el carácter dialéctico e histórico-cultural de este proceso, acompañante ineludible de toda la historia de vida. Tanto profesores como estudiantes universitarios deben tener conciencia sobre la importancia de la lectura no como instrumento de una determinada asignatura sino como eje que atraviesa la formación, el desarrollo y la educación del hombre a través de su existencia misma.

\subsection{Dimensiones curricular y extracurricular del proceso de formación para la promoción de lectura}

Estas dos vías constituyen dimensiones del proceso formativo concebido, pero están articuladas como modos de expresión del mismo; son dos formas de manifestarse este, que es único en cuanto a elementos que lo determinan, componentes estructuradores y principios dinamizadores, pero diverso en sus vías de expresión.

El desarrollo del proceso docente educativo revela la necesidad de establecer las relaciones entre sus niveles, básicamente la carrera, la disciplina y el año académico como soporte metodológico que da direccionalidad a la formación integral del profesional, asesorando otros niveles estructurales con un carácter inter y multidisciplinario, siendo estos más cercanos a la ejecución del trabajo didáctico. Dentro de estos niveles de interrelación en los currículos universitarios, se encuentran la Disciplina Principal Integradora y la llamada Estrategia Educativa del Año Académico.

Desde lo curricular no puede verse a la lectura unida a ninguna asignatura específica, sino que todas la presuponen. Las disciplinas de formación general, en sus relaciones de cooperación con la disciplina principal integradora deben establecer nexos con relación a la lectura por lo que el proceso deberá articularse de primero a quinto año de la carrera, y en él se identifican tres momentos o etapas, las que están determinadas por la estructura procedimental del método de promoción cultural.

Por lo que se infiere que la concepción que se pretende pueda ser implementada desde la Estrategia Educativa del año académico, pasando por tres etapas Diagnóstico, Desarrollo y Consolidación.

En las tres etapas todas las asignaturas y disciplinas tendrán presente el proceso según las características de sus contenidos y las potencialidades desarrollarlo.

Las etapas enunciadas en la dimensión curricular tienen su expresión correspondiente en la dimensión extracurricular.

Las actividades formativas para la dimensión extracurricular están contenidas también en la Estrategia Educativa del año académico como célula del trabajo metodológico de este colectivo, donde se concretan todas las acciones, tareas y actividades curriculares y extracurriculares que desarrollará el grupo o brigada estudiantil durante el curso escolar, en este sentido quedarán plasmadas las acciones de formación para la promoción de lectura.

Dichas acciones extracurriculares tienen su concreción más general en el trabajo científico estudiantil y en el trabajo sociocultural universitario. [Gestión de la extensión universitaria].
Tanto el trabajo científico estudiantil como el trabajo sociocultural universitario, tienen como escenario y vehículos apropiados el vínculo de los estudiantes con proyectos asociados a la lectura en la universidad y el territorio. A través de proyectos como forma de organización de la extensión universitaria se canalizarán las acciones, actividades y tareas extensionistas de formación para la promoción de lectura en los estudiantes.

La interdependencia entre lo académico, lo laboral y lo investigativo debe ser utilizada con la finalidad de potenciar progresivamente la relación entre lo curricular y lo extracurricular en la formación para la promoción de lectura.

En todo momento dichos abordajes deben garantizar la observancia de las leyes de la Didáctica, especialmente la que plantea [5] la necesaria relación entre los componentes no personales del proceso docente educativo; y la que postula la necesaria relación entre las dimensiones instructiva, educativa y desarrolladora.

En cuanto a la primera de las leyes debe ser tenida en cuenta para el desarrollo del proceso que nos ocupa, la articulación de los contenidos de la disciplina principal integradora con las disciplinas de formación general, partiendo de los puntos de cooperación entre esta asignatura y dichas disciplinas. Esta relación debe respetar en todo caso la adecuación de los componentes operacionales de la formación para la promoción de lectura a los fines esbozados en el objetivo como componente rector y al resto de los componentes de estado.

La relación entre las dimensiones curricular y extracurricular de la formación para la promoción de lectura se aprecia en la determinación de los contenidos comunes de las disciplinas de formación general desde la promoción de lectura, los cuales incluyen el sistema de conocimientos, habilidades, y los valores que deben ser abordados en una concepción pluridisciplinar.

Relaciones de tipo interdisciplinar entre las asignaturas de contenidos generales y las de formación para la profesión. Debe implicar una voluntad y compromiso de elaborar un marco más general en el que cada una de las disciplinas en contacto es a la vez modificada y pasan a depender claramente unas de otras.

Aquí se propone establecer una interacción desee la lectura entre dos o más disciplinas, lo que dará como resultado una intercomunicación y un enriquecimiento recíproco $\mathrm{y}$, en consecuencia, una transformación de las metodologías, una modificación de conceptos, de terminologías fundamentales.

Por su parte la coordinación de la formación para la promoción de lectura para las disciplinas básicas, básicas específicas y del ejercicio de la profesión debe ser articulada desde el trabajo metodológico de carrera, interdisciplinar, de disciplina y asignatura.

Esta coordinación debe tributar a un proceso formativo general de la profesión que tenga la función específica de incidir sobre el modo de actuación del futuro profesional que vea a la formación para la promoción de lectura como una forma de potenciar la lectura en los estudiantes, con protagonismo, con autogestión de su competencia lectora como esencial del ser humano para toda la vida.

Para ello debe orientarse la formación para la promoción de lectura del estudiante universitario partiendo de identificar problemas de la profesión susceptibles de ser trabajados desde 
la lectura entendiendo como problemas al conjunto de exigencias y situaciones inherentes al objeto de trabajo del profesional, que requieren de la acción del graduado para su solución.

Las relaciones de tipo transdisciplinar desde la formación para la promoción de lectura entre los contenidos al interior de las asignaturas de las disciplinas son de una trascendencia que supera el marco delimitador de una materia determinada.

Debe articularse como nivel superior de interdisciplinariedad, de coordinación donde desaparecen los límites entre las disciplinas y se constituye un nuevo sistema total que sobrepasa el plano de las relaciones e interacciones entre tales disciplinas.

En este tipo de relación con lectura debe atravesar de modo tal que como objeto propio, sin que esto se confunda, como hasta ahora, con una cadena de sucesiones de contenidos. La integración que se propone debe darse dentro de un sistema omnicomprensivo, en la persecución de unos objetivos comunes y de un ideal de unificación epistemológica y cultural.

La configuración necesaria para la integración de tipo transdisciplinar al interior de las asignaturas generales y en otras de formación para la profesión, supone la conformación de nuevos objetos de análisis, que se separen de la idea existente de concebir los contenidos como mezcla o sumatoria de manera arbitraria o aparentemente lógica.

Se propone entonces un marco de rediseño en cuanto al objeto de análisis de las disciplinas, y particularmente de los contenidos en ellas, cuya determinación se debe realizar mediante la abstracción de los campos de acción del profesional de una determinada carrera.

A lo anterior debe agregarse que la articulación del sistema de habilidades docentes en la formación para la promoción de lectura que debe partir de un ejercicio intelectual de abstracción de las competencias que distinguen las esferas de actuación del profesional de una determinada carrera.

Los componentes personales que en el proceso educativo universitario intervienen: profesores y estudiantes, son tenidos en cuenta desde la propuesta y deben asumir una actitud de cambio para poder interactuar y dar respuesta a los problemas presentes en el proceso que nos ocupa.

Es en el año es donde se logra la más íntima y cotidiana relación entre los elementos activos del proceso docente educativo, los estudiantes y los profesores: en él convergen de una forma u otra, distintas asignaturas que responden a distintas disciplinas enmarcadas en el plan de estudio de acuerdo con el modelo del profesional y que aparecen aquí en función del objetivo del año.

Así el colectivo del año académico desde el punto de vista del trabajo metodológico, incluye y concreta en él la relación con otros procesos que se dan a nivel de la Universidad, no solo la docencia sino también la extensión y la investigación.

La estrategia educativa desde dimensiones curricular y extracurricular y desde la que se aprecia también "la transversalidad del proceso extensionista” posibilita que la propuesta formativa que pretendemos se pueda desarrollar siempre sobre la base de las relaciones metodológicas del proceso docente educativo y bajo un fundamento que abarque desde las funciones didácticas de cada nivel hasta llegar al año académico como célula en la que se da la totalidad del trabajo metodológico en las universidades.

El Colectivo de Año por las posibilidades que tiene de concurrir en él todos los demás niveles del currículo y por el contacto más directo con los componentes personales de éste, tiene gran responsabilidad al tener como contenido básico el desarrollo de la personalidad de los estudiantes universitarios no solo en el orden de capacidades cognitivas, sino también axiológicas.

La relación entre los componentes del trabajo metodológico a nivel del año académico permite que la propuesta de formación para la promoción de lectura encuentre su expresión más pequeña del mismo pues en sí mismo refleja su cualidad totalizadora e impide, a partir de esto, el desmembramiento en partes de ese trabajo, al mismo tiempo que integra todos los componentes y funciones. El año académico constituye la célula del trabajo metodológico en las universidades y es precisamente ahí donde se inserta nuestra propuesta.

En el desarrollo del proceso de formación para la promoción de la lectura en la Estrategia Educativa del año, el estudiante juega un papel aún más protagónico, en tanto se relaciona aquí con un mayor número de elementos confluentes de éste, la carrera y su modelo de profesional, las disciplinas docentes y su accionar instructivo-educativo en relación con la elevación técnica y metodológica del mismo, a través de las diferentes asignaturas; la relación entre profesores y estudiantes es más estrecha y directa y con un accionar más colectivo, los procesos universitarios de docencia, extensión e investigación tienen aquí un campo de relaciones más sólido.

De esta manera [6] expresa que los aspectos a desarrollar por cada uno de los componentes del proceso de formación para la promoción de lectura deben estar presentes y ligados en cada momento tanto a objetivos, contenidos y sistema de actividades y evaluación, mediante "una metodología más interdisciplinar y contextualizada.

El desarrollo del proceso de formación para la promoción de lectura, derivado de relaciones del trabajo metodológico a través del año académico es factible si se integran y evalúan las acciones metodológicas encaminadas a este desde lo curricular y extracurricular con el fin de lograr una estrategia acorde con una sólida unidad de lo educativo y lo instructivo y lo desarrollador.

La transversalidad asociada a la lectura propone flexibilizar fundamentalmente los métodos, las formas y la evaluación del aprendizaje, por el carácter dinámico que poseen dichos componentes en el proceso de enseñanza - aprendizaje.

Desde lo curricular, la lectura como elemento integrador en el programa de las asignaturas, exige una mayor apertura y flexibilidad en el diseño y desarrollo curricular, abriendo las compuertas entre disciplinas que hasta ahora han sido entendidas de modo cerrado e independiente.

Para lograrlo, cobra especial importancia el papel que desempeñan los proyectos, actividades, acciones y tareas extensionistas de formación para la promoción de lectura que puedan desarrollarse. Dichas tareas no han de plantearse como ejercicios o actividades al modo tradicional, sino como zonas de intersección entre disciplinas, como puentes que faciliten al alumnado darse cuenta de la conexión intrínseca entre los diferentes contenidos desde la promoción. 
Esta reforma metodológica tiene, lógicamente, algunas repercusiones en los componentes personales del proceso. Sólo indicaremos brevemente una de ellas, vista desde ambos lados de la relación didáctica:

- Propicia un cambio en el rol del profesor como mediador. Ello le exigirá un mayor esfuerzo de preparación de tareas e integración de aprendizajes, además de tener que vencer la resistencia a los cambios tanto en la planificación curricular como en la práctica.

- Promueve, de modo necesario, una mayor actividad en el estudiante, pues es una forma de incorporarse en el proceso de enseñanza-aprendizaje de una manera más dinámica y flexible.

Con una metodología basada en la promoción, los estudiantes pueden implicarse de una forma más protagónica. La implicación que supone el desarrollo del proceso a través de diversas tareas, facilita que el aprendizaje sea más significativo $\mathrm{y}$, por tanto, la motivación mayor al advertir un avance en el conocimiento.

Desde una dimensión extracurricular es preciso reflexionar y profundizar en un cambio en el modo de trabajar más allá de la metodología empleada en el aula. Ésta, para tener éxito -dada su complejidad- necesita el soporte formativo, personal e institucional que haga posible el profundo cambio metodológico aludido.

La formación individual es necesaria para el proceso de formación para la promoción de lectura, pero esta precisa de un planteamiento institucional que no sólo permita, sino que fomente una mayor comunicación profesional docente con relación a la lectura.

El cambio ha de ir orientado hacia la creación de equipos docentes en los que, mediante el trabajo cooperativo entre los profesores del año y diferentes áreas de la universidad, se facilite la propuesta de actividades y tareas extensionistas interdisciplinares que ayuden a desarrollar el proceso de forma integrada.

La transmisión y comunicación de experiencias en el colectivo de año puede permitir una mayor implicación en las tareas extensionistas, lo que facilitará necesariamente un aumento de la motivación y una conducta lectora adecuada. Así, este planteamiento extensionista interdisciplinar desde lo extracurricular es en sí mismo, formativo.

Es por eso que el proceso que pretendemos no puede estar desvinculado del proceso formativo que se desarrolla en la universidad sino en estrecha relación, la enseñanza no puede separar lo cognoscitivo de lo desarrollador.

El profesor como principal gestor del proceso de formación para la promoción de lectura, asume su preparación como función extensionista relacionada con la lectura teniendo en cuenta la pertinencia de:

- Determinar las necesidades de aprendizaje en correspondencia con las necesidades sociales y relacionarlas desde la promoción de la lectura para el perfeccionamiento y la elevación del nivel cultural y educacional de sus estudiantes.

- Indagar sobre las prácticas lectoras de sus estudiantes en los diferentes escenarios de actuación y los niveles de competencias alcanzados con relación a la lectura.
- Revisar planes de estudio y las áreas de contenidos a considerar donde pueda promoverse de forma integrada la lectura.

- Explorar la percepción de sus estudiantes en relación con el deber ser en su labor cotidiana y como coordinador del proceso.

- Investigar sobre prácticas formativas similares y espacios universitarios que permitan la potenciación del proceso.

- Identificar el potencial institucional (comunidad universitaria) e integrar al proceso de formación para la promoción de lectura.

- Determinar necesidades de todo tipo que imposibiliten el mejor desenvolvimiento del proceso.

- Evaluar las condicionantes capaces de favorecer el proceso de formación para la promoción de lectura.

El profesor facilita una relación activa entre el estudiante y la lectura, teniendo en cuenta en este proceso la autogestión, la participación y la integración a las instituciones que tiene a la lectura como principal (instituciones lectoras) dentro y fuera de la universidad.

El profesor como mediador del proceso debe ser capaz de relacionarse y establecer un intercambio recíproco con las instituciones lectoras evitando actuar de forma aislada y propiciando la interacción con el resto de los agentes sociales que de una forma u otra inciden en la comunidad: bibliotecarios, libreros, y personal de instituciones afines.

En criterio de [7] la transversalidad curricular se manifiesta en dos direcciones:

I- A través de su influencia, al flexibilizar el sistema de componentes didácticos del proceso docente educativo en cada nivel de su estructuración (nivel, carrera, disciplina, asignatura, clase, tema, tarea docente), en función del desarrollo de los elementos para asumir con éxito las acciones, lo que implica tener en cuenta:

- El trabajo en equipo del colectivo pedagógico, para ejercer un sistema de influencias educativas

- La disposición, motivación y preparación del colectivo pedagógico para hacerlo.

- El reconocimiento por parte de los profesores, empleadores y estudiantes de la necesidad del desarrollo del proceso.

- La aplicación de métodos problémicos y de proyectos, en el desarrollo de las actividades docentes, investigativas y laborales.

- Lograr el fomento del clima pedagógico en el desarrollo de todas las actividades que se realizan en el currículo.

- El reconocimiento en el proceso de enseñanza - aprendizaje de la diversidad para potenciar el desarrollo de cada estudiante, de acuerdo con sus características y potencialidades y sin perder de vista el encargo social en el que reflejan las aspiraciones de la sociedad respecto a la formación ciudadana.

- Implica la toma de conciencia del colectivo pedagógico y de los estudiantes de estar inmersos en un proceso de formación.

- II- A través de la integración de conocimientos, habilidades, capacidades y valores adquiridos en las disciplinas y asignaturas de cada año académico, lo que implica: 
- La integración de la formación académica, laboral e investigativa en las actividades relacionadas con la profesión y con el proceso de formación.

- Vincular los problemas presentes en la práctica social al proceso docente educativo, con el objetivo de retroalimentar el desarrollo del proceso y en función de ello; corregir dificultades y movilizar las necesidades, motivaciones e intereses de los estudiantes.

- Responsabilizar a los profesores con el proceso de formación.

Calichs teoriza que "La articulación necesaria entre los objetivos, contenidos, métodos, formas, medios y evaluación con el desarrollo de los elementos necesarios (...), para propiciar, como punto de partida del proceso de formación, los modos de conocimientos asociados a la actuación del profesional; de manera que el estudiante, con un mayor nivel de definición, pueda otorgar un significado a lo que aprende y relacionarlo con lo laboral".

\subsection{La metodología de la promoción sociocultural en el proceso de formación para la promoción de lectura}

Comprende además del conocimiento del contexto, el papel del mediador, es decir, el docente, de los estudiantes, sus concepciones ideológicas, identidad, sentimientos y estados de ánimo, pertenencia a un grupo social, su intención y finalidad promocional.

Entre los factores que condicionan la promoción cultural se destacan las instituciones culturales que desde el enfoque de la dirección científica de los procesos culturales, se conciben como medios necesarios para la dinamización de la vida cultural. Estas poseen entre sus propósitos acrecentar los intereses culturales de la población, aunar un público activo y constante, y promover los más altos valores artísticos a escala nacional e internacional.

La promoción sociocultural es el conjunto de tareas encaminadas a estimular el vínculo activo cultura-sociedad de manera que se logre elevar el desarrollo en ambas, pues es este el medio idóneo para alcanzar niveles superiores en el desarrollo sociocultural y dar respuesta a las necesidades siempre crecientes de la población.

La promoción sociocultural aparece en escena por la necesidad de promover el hecho cultural en la sociedad, colocando en movimiento un proceso a través del cual se crean condiciones favorables para que los grupos sociales y las comunidades encuentren sus propias respuestas y expresen con toda libertad su sentido de pertenencia e identidad.

Coincidimos con [8] cuando emite un concepto de promoción sociocultural abarcador e integrador donde en un sentido más estrecho, se concibe como mecanismo de estímulo y motivación en el hombre, dadas las formas utilizadas, para hacerlo partícipe de la cultura, a través de la animación y la información. Además, desde el punto de vista metodológico permite activar las comunidades, barriadas y pequeños poblados, generando procesos participativos.

La promoción sociocultural, según fue expresado por [9], es un proceso que lleva implícito un cambio en la forma de ser y actuar del hombre, reúne un sistema de dirección, planificación, organización, administración, seguimiento y control, una metodología y mecanismos de estímulos tales como la propaganda, la publicidad, la divulgación y relaciones públicas, entre otras.

El Modelo de Gestión para la Extensión Universitaria en la Universidad Cubana fundamentado desde las ciencias pedagógicas por [10] asume como metodología de la extensión a la promoción cultural, al ser este un proceso formativo que por su forma y contenido permite su expresión dentro y fuera de la universidad y se establece desde la comunicación, y la actividad.

La concepción que pretendemos no puede estar aislada del modelo extensionista de gestión por lo que se establece desde la promoción cultural, determinando que la promoción de lectura es promoción cultural y poniéndose de manifiesto en ella los principios y leyes generales de la extensión universitaria.

Estos principios planteados como aquellos que dinamizan y regulan la concepción pedagógica propuesta, asumen los rasgos característicos del objeto que se modela: el proceso de formación para la promoción de lectura en la extensión universitaria, tienen en cuenta las falencias que dieron origen a la investigación, las bases teóricas asumidas, el análisis tendencial del objeto de investigación, así como las regularidades del comportamiento del objeto en el contexto analizado y la naturaleza pedagógica de la concepción, de sus principios y de las etapas.

Estos principios reflejan en su esencia, las bases científicas del proceso de formación para la promoción de lectura en la Universidad, la lógica de la concepción pedagógica, el enfoque integral del proceso durante todas las etapas, que coinciden con los ciclos de formación universitaria, las contradicciones, interacciones y relaciones que se producen durante el mismo, $\mathrm{y}$ la formación transversal de la promoción de la lectura, de tal manera los principios son expresión no solo de las características esenciales, sino de las necesarias al proceso formativo estudiado.

La metodología de la promoción cultural vinculada al proceso formativo genera las habilidades que necesita el estudiante para desarrollar. La lectura, acerca al estudiante a su realidad profesional y al cumplimiento de sus funciones como futuro profesional.

De este modo, el estudiante en su formación, desarrolla un conjunto de habilidades desde la promoción de lectura que lo preparan mejor para su desempeño profesional y personal y lo convierte en multiplicador. Es por eso que el proceso de formación para la promoción de lectura, tiene que ser entendido desde una perspectiva científica.

El proceso de formación para la promoción de lectura, debe entenderse como un proceso que facilite al promotor- lector apropiarse de una conducta lectora y que desde el proceso formativo, el método de la promoción cultural, los principios que la rigen y sus relaciones permita reconocer científicamente los problemas de propios de su profesión así como un mejor entendimiento de la sociedad en que se desarrolla.

La relación entre el proceso formativo en la extensión universitaria como un proceso sustantivo que tiene como fin la promoción de la cultura se articula a partir de conceptos, principios y componentes que sirven de base al proceso que pretendemos, el que se integra de manera armónica dentro del 
otro más general. La gestión del proceso extensionista por su naturaleza y características, revela la dialéctica de lo general, lo particular y lo específico.

Desde lo general la extensión se articula coherentemente en la planeación estratégica universitaria y la dirección por objetivos, tributando a partir del trabajo sociocultural universitario a los valores compartidos de la universidad; en lo particular, desde su dimensión administrativa, asume las peculiaridades propias de la extensión y se mueve a partir de las funciones de dirección y en lo específico la gestión se materializa desde los métodos propios de la promoción sociocultural, definida como metodología de la extensión..

En este sentido el proceso de formación para la promoción de lectura en la extensión universitaria se visualiza la relación dialéctica de lo general, lo particular y específico y se instrumenta en la práctica del Trabajo Sociocultural Universitario asumiendo la metodología propia de la extensión.

\section{Etapas del proceso de formación para la promoción de lectura}

La formación para la promoción de lectura es un proceso porque evoluciona, se desarrolla y avanza en el tiempo a través de tres etapas sucesivas, conducentes a lograr como resultado cambios en el proceso de formación profesional, a través de la incidencia en sus componentes personales. Comienza con la familiarización de los estudiantes, en la cual a través de la aplicación de las diferentes técnicas, se determina el comportamiento de los indicadores que pudieran tener mayor incidencia en su proceso de formación como profesionales. Esta etapa incluye un pronóstico, atendiendo a las fortalezas, debilidades, oportunidades y amenazas detectadas a partir del procesamiento de los datos, que permite a los coordinadores formular predicciones futuras teniendo en cuenta la zona de desarrollo próximo en cada caso.

La etapa de desarrollo requiere la capacitación constante de los mediadores, con vistas a que sus acciones de obtengan en los estudiantes el efecto positivo deseado.

El proceso docente educativo es un espacio ideal y la clase puede convertirse en el principal espacio para el proceso de formación para la promoción de lectura. De igual forma los profesores, capacitados como coordinadores y con la información aportada en el proceso de familiarización de sus estudiantes, estarán en condiciones de desarrollar de conjunto actividades, acciones y tareas extensionistas como parte de dicho proyecto extensionista.

La consolidación se convierte en una etapa fundamental para revisar los resultados alcanzados a través de la coordinación de actividades, acciones y tareas extensionistas de formación para la promoción de lectura, permite la sistematización de coordinadores y multiplicadores del proceso, repetir algunas técnicas que han ofrecido resultados favorables, corregir otras que no han tenido buena aceptación y/o repercusión en los estudiantes.

\section{Principios en los que se sustenta del proceso de formación para la promoción de lectura en la extensión universitaria.}

A partir del estudio teórico efectuado, la concepción pedagógica del proceso de formación para la promoción de lectura en la extensión universitaria, se desarrolla sobre la base de los siguientes principios, que funcionan como reguladores y dinamizadores de este proceso:

- Carácter transdisciplinar.

- Carácter contextual.

- Carácter creativo

- Carácter sociocultural.

\subsection{Carácter transdisciplinar}

Hablar de transdiciplinariedad de un proceso de formación para la promoción de lectura significa referirse a otra forma de entender y organizar el aprendizaje a partir de la lectura en el contexto universitario. Ello conduce, necesariamente, hacia la utilización de nuevas estrategias metodológicas y necesariamente formas de organización de los contenidos.

De esta manera se propicia la formación de futuros profesionales autónomos, críticos, con criterios propios y capaces de hacer frente a los problemas socioculturales que tiene planteados hoy la humanidad.

El concepto de transdiciplinariedad usado de diversas maneras, desde la necesidad de plantear formas de investigación integradoras a la necesidad de crear espacios nuevos para una investigación más allá de las disciplinas.

En sus estudios [11] plantea un enfoque multidisciplinar relacionado con el abordaje de un tópico de investigación desde varias disciplinas simultáneamente, en el enfoque interdisciplinarse produce habitualmente la transferencia de unos métodos de análisis de una disciplina a otra. En ambos casos, la indagación permanece en los ámbitos de las disciplinas ya configuradas, en un solo nivel de realidad, mientras que en el caso de la investigación transdisciplinar se reubica en las dinámicas entre los diversos niveles de la realidad.

El enfoque transdisciplinar es necesario cuando abordamos el estudio del ciberespacio o la cibercultura. Es decir, el enfoque transdisciplinar cobra sentido en situaciones de gran complejidad, como ocurre precisamente con todo lo que es el mundo de la lectura.

La transdiciplinariedad es radicalmente diferente de la multidisciplinariedad y de la interdisciplinariedad debido a su objetivo, la comprensión del mundo actual en su complejidad, que no puede alcanzarse en el entramado de los estudios disciplinares. Y agregan que cuando se trata de conocer las prácticas culturales de lectura en un entorno concreto, el objetivo no puede limitarse a una investigación disciplinar, de carácter sociológico, educativo, etc.

En el caso de la propuesta que nos ocupa se hace necesario romper esquemas que crean barreras entre disciplinas y, trabajar desde la promoción y la creatividad para unificar conceptos, métodos o datos que existen en disciplinas distintas para crear una síntesis nueva.

El principio de la transdiciplinariedad referido a la extensión radica en garantizar un enfoque multi y 
transdisciplinar en la solución de los problemas identificados como necesidades sentidas del propio colectivo o de otros colectivos de la universidad o de fuera de ella, materializando una estrecha relación entre los tres procesos sustantivos, en función de incentivar la formación del educando desde una perspectiva cívica y de vocación social, irrenunciable en el contexto formativo del país y tributar a la propia transformación de la Universidad.

En este sentido el proceso de formación para la promoción de lectura es transdisciplinar porque no se enfoca en disciplina determinada, sino que todas la presuponen.

Al mismo tiempo no excluye la utilización de mecanismos interdisciplinares desde las formas organizativas del proceso docente fundamentalmente el colectivo de año quien puede fomentar relaciones interdisciplinares a favor de la formación para la promoción de lectura.

\subsection{Principio del carácter contextual}

El contexto es definido por la Real Academia de la Lengua Española, en una de sus acepciones como "Entorno físico o de situación, ya sea político, histórico, cultural o de cualquier otra índole, en el cual se considera un hecho.” Es el entorno en el que transcurre cualquier hecho o acontecimiento que generalmente incide o influye en su desarrollo según el Diccionario de las Ciencias de la Educación todas las organizaciones se encuentran inmersas en un determinado contexto que condiciona su desarrollo. La concepción que pretendemos debe considerar el contexto en el cual se desarrolla.

Así como los múltiples factores políticos, económicos, tecnológicos, etc.; que influyen con diferente nivel de impacto tanto dentro como fuera de la universidad

Este principio se basa en uno general de la extensión universitaria y también de la promoción de lectura.

Por lo tanto, no puede sustraerse el contexto del proceso de formación para la promoción de lectura, este es determinante porque condiciona dicho proceso.

El sistema de influencias contextuales en el que el estudiante universitario se desenvuelve es de gran diversidad. En él influyen diversos factores externos e internos que están presentes en el marco institucional como fuera de los recintos universitarios por lo que se requiere potenciar recursos personales para que movilicen sus potencialidades y superen las limitaciones, lo cual refuerza la necesidad tener en cuenta el contexto.

El contexto resulta diagnosticable, diseñable construible y utilizable para las personas que protagonizarán los procesos que en él se desarrollen.

El contexto resulta oportunidad susceptible de indagación, como interfaz dinámica e inestable.

\subsection{Principio del carácter creativo}

El trabajo sociocultural articulado desde la promoción cultural supone, como principio, la incorporación y participación voluntaria a partir del desarrollo de intereses y motivaciones.

Para concebir el desarrollo de la creatividad, resultan de gran valor los trabajos iniciados en los años 1950 por el profesor Guilford, pues la consideró como una forma de pensar, donde la sensibilidad a los problemas constituyen el punto de partida; los estudios realizados por el psicólogo. La concepción teórica de Albertina Mitjáns [1995] sobre el carácter personológico de la creatividad, al definirla como una expresión de la personalidad asociada a importantes indicadores y expresiones funcionales de ella.

Existen tantas definiciones como puntos de vista y posiciones teóricas diferentes, lo que coloca al investigador frente a un fenómeno polisémico o de significación plural. La creatividad extiende sus ramificaciones al ámbito neurológico, psicológico, organizativo, pedagógico, artístico, técnico, humanístico y científico.

A juicio de [8] los siguientes aspectos no deben omitirse en la concepción de creatividad en los tiempos actuales:

- "Motivaciones significativas: Porque el proceso para potenciar el desarrollo de la creatividad está relacionado con la presencia de formaciones motivacionales complejas las que explican la conducta creativa en las personas y la profesión es una tendencia orientadora y motivacional de la personalidad. La creatividad también se expresa allí donde el sujeto está significativamente implicado.

- Experiencia acumulada: Porque constituye un elemento extrapersonal que permite incrementar las motivaciones reales, en dependencia del conocimiento científico o desarrollo tecnológico existente en un momento dado.

- Capacidades logradas: Porque aunque la inteligencia no es sinónimo de creatividad esta última se desarrolla a partir de las habilidades y capacidades desarrolladas en el proceso cognitivo. Aplicar las capacidades logradas y necesarias ante una nueva situación, garantiza obtener resultados creativos.

- Personalidad del sujeto: Porque el desarrollo de la creatividad no puede darse al margen de la personalidad del individuo; La creatividad es una expresión de la personalidad en su función integradora, donde intervienen las motivaciones, los procesos cognitivos, afectivos y volitivos.

- Oportunidades y posibilidades: Porque las dimensiones social y cultural de la creatividad hacen que su desarrollo dependa también de las oportunidades que brinda el contexto, a partir de su desarrollo sociohistórico y de las posibilidades que brinde al individuo de potenciar el proceso creativo.

- Un producto original y relevante tangible o no: Porque de alguna manera, para evaluar el desarrollo creativo es necesaria la presencia de un resultado tangible o no, original y relevante, lo que implica la solución del problema.

- Competencia creativa: Porque constituye el elemento indicador por excelencia de la calidad en el desempeño profesional y los niveles alcanzados en el desarrollo creativo.

\subsection{Principio del carácter sociocultural}

El principio del carácter sociocultural implica que el proceso de formación para la promoción de lectura en la universidad. 
Lo sociocultural como su nombre indica una realidad vista desde ambos campos, lo social y lo cultural, lo que en criterio de [12] convendría hablar más bien del enfoque sociocultural de los procesos sociales y culturales. Lo que es bien cierto es que existe una interdependencia entre ambos campos.

El principio del carácter sociocultural del proceso que nos ocupa se basa en el entendimiento de que lo social y lo cultural si bien son realidades relativamente independientes se interrelacionan específicamente teniendo en cuenta un contexto histórico determinado.

Los desafíos de la extensión universitaria son esencialmente culturales y la solución radica en el fomento de la cultura, tarea encomendada de forma explícita a la universidad.

La extensión universitaria asume hoy su propia concepción de preparar y consolidar las universidades como instituciones de cultura y la ampliación de la influencia recíproca e integración con la sociedad mediante la promoción de la cultura en el sentido más amplio, humanístico, científico, técnico, profesional y deportivo.

El Programa Nacional de Extensión Universitaria para la Educación Superior cubana, surgido en abril de 2004, fue concebido con la intención de convertirse en un instrumento eficaz para la gestión del proceso extensionista en las universidades.

Se concibió para que la universidad como institución cultural contribuyera desde promoción sociocultural en la comunidad intrauniversitaria y la de su entorno.

\section{Principales resultados}

A partir de la concepción presentada, se diseñó una estrategia para su implementación en las carrera de ingeniería a través de tres acciones estratégicas específicas, de las cuales resultaron como productos: Programa de Promoción de Lectura en la Extensión Universitaria; el Programa de capacitación para los docentes, Sistema de talleres extensionistas de formación de promotores estudiantiles de lectura, así como el Observatorio Universitario de Promoción de la Lectura. Para evaluar la factibilidad y validez de la concepción y de la estrategia diseñada, se utilizó el método de criterio de expertos, que aportó resultados devenidos en importante contribución al perfeccionamiento de esta.

\section{Conclusiones}

En la actualidad se le atribuye gran interés a la formación para la promoción de lectura, la que constituye un tema actual ligado al desarrollo intelectual, espiritual y social de los individuos. Un proceso dirigido a este fin en la universidad dentro de una propuesta integrada y sistémica en la extensión universitaria que contribuya al perfeccionamiento de esta función sustantiva y de la Educación Superior en general, constituye una necesidad, dada la importancia de preparar a los futuros profesionales para su desarrollo en una sociedad en constante transformación.

El proceso de formación para la promoción de lectura no constituye objetivo en la extensión universitaria, se concreta solo a acciones y actividades extensionistas aisladas, desconocimiento de las potencialidades de la promoción cultural, no se tiene en cuenta el tratamiento de la lectura con un fin en sí misma, ni el entendimiento de las nuevas formas de lectura, por lo que se ejecuta de manera espontánea, escasa y asistémica.

La concepción pedagógica propuesta se reconoce como el conjunto de ideas científicamente argumentadas que permiten la determinación de los fundamentos pedagógicos del proceso de formación para la promoción de la lectura, la que se sustenta en la extensión universitaria y su método la promoción sociocultural en las dimensiones curricular y extracurricular, a partir del método de la promoción cultural, estructurada en las etapas de familiarización, desarrollo y consolidación, y dinamizada por los principios del carácter contextual, transdisciplinar, creativo y sociocultural de dicho proceso.

\section{Referencias}

[1] Ramírez-Leyva, E.M. et all., Tendencias de la lectura en la Universidad. Compilación. Instituto de Investigaciones Bibliotecológicas y de la Información. [en línea]. 2015. Recuperado de: http://iibi.unam.mx/publicaciones/292/01_tendencias_lectura_universid ad\%20_elsa_ramirez_leyva.html

[2] Bombini, G., Sabemos poco acerca de la lectura. Lenguas Vivas, Publicación del Instituto de Enseñanza Superior Juan Ramón Fernández, 2(2), octubre-noviembre, Buenos Aires, 2002.

[3] Hernández-Guzmán, Y., Metodología para la autogestión de la formación de los estudiantes universitarios. Su aplicación en la Universidad de Pinar del Río. Tesis de MSc., Centro de Estudios de Ciencias de la Educación Superior. Universidad de Pinar del Río, Cuba. 54 P., 2015.

[4] Gil-Flores, J., Hábitos lectores y competencias básicas en el alumnado de educación secundaria obligatoria. Universidad Nacional de Educación a Distancia. Madrid. Educación XX1, 14(1), pp. 117-134, 2011.

[5] Chávez-Rodríguez, J., La investigación científica desde la escuela. En desafío escolar. Revista Iberoamericana de Pedagogía. 6(Ed. Especial), ICCP, Centro de Investigación y Desarrollo Educacional. Cuba- México. 2001.

[6] Álvarez-de Zayas, C. y Sierra, V., La investigación científica en la sociedad del conocimiento. Material de apoyo a la docencia. La Habana, Cuba, 2002.

[7] Martos-Núñez, E., De la República de las letras a Internet: De la ciudad letrada a la cibercultura y las tecnologías del S. XXI. Revista Álabe. [En línea]. 1 jun, 2010. Recuperado de: http://revistaalabe.com/index/alabe/article/view/0/6

[8] López, E., Modelo para el proceso de formación de las competencias creativas en los estudiantes de la carrera de Licenciatura en Estudios Socioculturales de la Universidad de Pinar del Río. Tesis Dr. en Ciencias Pedagógicas. CECES. Universidad de Pinar del Río. Cuba, 2005.

[9] Fernández-Larrea-González, M., Un modelo de gestión del proceso extensionista en la Universidad de Pinar del Río. Tesis Dr. en Ciencias de la Educación, Universidad de Pinar del Río, La Habana, Cuba, 2004.

[10] González-González, G. y González-Fernández-Larrea, M., La gestión de la extensión universitaria desde la perspectiva cubana. Revista Cubana de Educación Superior, 2, La Habana, 2004.

[11] Freire-Roach, E., ¿Qué son los estudios socioculturales? En: Lo sociocultural un trabajo pendiente. Rojas, B. y Rodríguez, L.A. (Comp.), La Habana. Editorial de Ciencias Sociales, Cuba, 2013

[12] Yáñez-Pérez, L., En lo sociocultural un trabajo pendiente. Comp: Rojas, B. y Rodríguez, L.A. (Comp.), La Habana. Editorial de Ciencias Sociales, Cuba. 2013.

Y. Rovira-Álvarez, recibe el título académico de Lic. en Educación en la Especialidad de Español y Literatura en el año 1995. MSc. en Desarrollo Social en el año 2005 en la Facultad Latinoamericana de Ciencias Sociales (FLACSO) de la Universidad de La Habana. Cuba. Recibe el título académico de MSc. en Ciencias de la Educación en el año 2015 en el Centro de Estudios de Ciencias 
de la Educación Superior (CECES) de la Universidad de Pinar del Río, Cuba. Ha laborado en el Departamento de Extensión Universitaria de la Vicerrectoría de igual nombre de la Universidad de Pinar del Río, Cuba desde el año 2000, donde se desempeña como profesora.

ORCID: 0000-0003-3232-9372

E. López-Calichs, recibe el título académico de MSc. en Ciencias de la Educación en el año 2001 y Dr. en Ciencias Pedagógicas en el año 2004. Todos ellos en la Universidad de Pinar del Río, Cuba. Ha laborado en el Centro de Estudios de Ciencias de la Educación Superior (CECES) de la Universidad de Pinar del Río, Cuba desde el año 2000. Se desempeña como metodólogo de posgrado de dicho centro.

ORCID: 0000-0005-4537-8394

A. Jiménez-Morejón, recibió el título de Ing. Mecánico en 1997 y el de MSc. en Desarrollo Social en 2013, todos ellos de la Universidad "Hermanos Saíz Montes de Oca”, Pinar del Río, Cuba. De 1997 a 2005 trabajó para compañías constructoras y del sector automotriz cubano. Se vinculó a la Universidad "Hermanos Saíz Montes de Oca” de Pinar del Río, Cuba en el año 2006 y es profesora asistente desde el año 2012. Ha trabajado en programas y proyectos de trabajo sociocultural comunitario del área de extensión universitaria, con énfasis en la gestión y formación de los artistas aficionados universitarios. Actualmente es profesora del Departamento Docente de Extensión Universitaria.

ORCID: 0000-0003-5547-9232 